\title{
Transvesical Endoscopic Port in Abdominal Surgery: An Updated Perspective
}

\author{
Estevao Lima • Riccardo Autorino - Jorge Correia-Pinto
}

Published online: 23 February 2010

(C) Springer Science+Business Media, LLC 2010

\begin{abstract}
Natural orifice transluminal endoscopic surgery (NOTES) generated a huge hope among surgeons because it promised scarless surgery and eventually less pain and surgical stress. However, serious limitations regarding reliable visceral closing methods remain unsolved. This article provides an update in development and future applications of transvesical access in the field of surgery.
\end{abstract}

Keywords Transvesical surgery · NOTES .

Minimally invasive surgery $\cdot$ Laparoscopy

\section{Introduction}

Since the introduction of laparoscopic surgery in the early 1990 s, it has been shown that reduction of surgical incisions reduces postoperative pain and recovery time. Envisioning evolution, Reddy and Rao from India performed a very controversial procedure: human transgastric appendectomy $[1 \bullet \bullet]$. Recognizing the potential benefits of this procedure, Kalloo et al. [2••] in 2004 pursued this idea, in a very well-structured way, by testing in pigs the feasibility and limitations of abdominal surgery without scars through a transgastric port. This was the birth of natural orifice transluminal endoscopic surgery (NOTES). This approach was envisioned with several advantages once it would avoid the abdominal incisions and all the consequences of them, such as incisional hernias and infections of surgical wounds. Moreover, it has the theoretical potential to further reduce the postoperative

\section{E. Lima $\cdot$ R. Autorino $\cdot$ J. Correia-Pinto $(\triangle)$}

Life and Health Sciences Research Institute (ICVS),

School of Health Sciences, University of Minho,

Braga, Portugal

e-mail: jcp@ecsaude.uminho.pt pain and recovery time when compared with laparoscopy $[1 \bullet \bullet]$.

Subsequently, various authors described more complex intra-abdominal NOTES procedures. After these experimental procedures, there was a terrific debate about the potential benefits from transgastric access and the several challenges/limitations being identified by the groups who were testing the transgastric port [3]. In fact, there was a consensus that the transgastric access was not totally sterile, and the difficulties in its endoscopic closure could be the cause of serious complications in abdominal surgery. Despite the aggressive criticisms from the most conservative surgeons and endoscopists, the possibility to perform scarless surgery nursed an increasing number of dreamers and believers in NOTES. Thus, a joint effort by key persons from the American Society for Gastrointestinal Endoscopy and the Society of American Gastrointestinal Endoscopic Surgeons collected the preliminary data and summarized in a white paper the most important limitations and some potential strategies to overcome them $[1 \bullet \bullet]$. The major limitations of isolated transgastric port were related to the nature of the gastroscope instruments (flexible and parallel), which caused the surgeons to lose some important principles from classical and laparoscopic surgery during transgastric procedures, including absence of triangulation, poor retraction capability, and the necessity to work

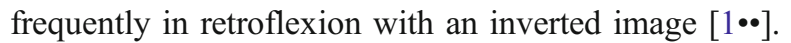

The early history of minimally invasive surgery in urology is unknown to many surgeons, but the first attempts to explore the natural orifice of the human body go back a long time. In 1804, Philip Bozzini developed a light conductor called the "Lichtleiter" and attempted direct bladder inspection [4]. Almost a century later, Boisseau de Rocher in 1890 introduced a mignon lamp cystoscope that offered the first double channel for ureteral catheterization 
[4]. This was the foundation of a new era using the transurethral route as a way to perform therapeutic endoscopy in the urinary system. In fact, this pathway allowed the treatment of much pathology of both lower urinary tract (benign prostatic hyperplasia and bladder tumors) and, recently, high urinary tract (urothelium tumors and lithiasis) using semirigid or flexible ureterorenoscopes. Until recently, however, it was never intended to use transurethral/transvesical approaches in surgery outside of the urinary tract. Indeed, Lima et al. [5・•] were the first authors whom in 2006 predicted the potential application of the transvesical approach and foreseen the bladder as a viable portal of entry for abdominal and thoracic surgery. Using current urologic instruments, our research group reported an atraumatic method to create a transvesical port.

\section{Transvesical Access Technique}

The placement of the transvesical port is based on the Seldinger principle [5••]. Currently, a ureteroscope is introduced through the urethra into the bladder with pneumodistension, emptying urine from the bladder and distending it with $\mathrm{CO} 2$. The vesicotomy site is carefully selected on the bladder dome. A mucosal incision is made with scissors introduced through the working channel of the ureteroscope. Subsequently, a five Fr open-ended ureteral catheter is pushed forward through the incision into the peritoneal cavity. A 0.035 -inch flexible tip guidewire is then inserted into the peritoneal cavity through the lumen of the ureteral catheter. Guided by the flexible tip guidewire, the vesical hole is enlarged with a dilator of a ureteroscope sheath enveloped by a flexible $5.5-\mathrm{mm}$ overtube. A ureteroscope is introduced into the peritoneal cavity through the overtube and allows the creation of a pressure-controlled $\mathrm{CO}_{2}$ pneumoperitoneum. The ureteroscope is ultimately withdrawn from the abdominal cavity after $\mathrm{CO} 2$ removal, and a Foley catheter is inserted into the bladder for 3 and 4 days. Recently, Lima et al. [6] reported for the first time a method for endoscopic closure of transmural bladder wall perforations.

\section{Experimental Work Using the Transvesical Port}

The first documented case of surpassing the bladder wall to perform simple intraperitoneal procedures was carried out and reported by Lima et al. [5••]. The authors introduced a transurethral and transvesical ureteroscope into the peritoneal cavity, and subsequently, liver biopsy and division of the falciform ligament were performed. Postoperatively, the survival animals were left with a catheter for 4 days, after which necroscopy revealed completely healed cystotomy sites and no evidence of peritoneal complications.

Given the unexpected good results from the first study using transvesical port, Lima et al. [7] felt encouraged to test the possibility to reach even the thoracic cavity. A ureteroscope was introduced into the peritoneum through the transvesical port and was subsequently advanced into the thoracic cavity. The insufflation was achieved through the ureteroscope, and lung biopsies and inspection of the pleural cavity and lung surface were performed with success. A Foley catheter was left in the bladder for 4 days, and the postmortem examination 15 days after surgery revealed complete healing of the vesical and diaphragmatic incision. Although the authors had been able to perform only limited thoracoscopy and lung biopsies, it definitively extended the intervention field of NOTES from peritoneal to thoracic cavity as well.

Cholecystectomy is one of the most challenging isolated transgastric approaches. Using two endoscopes, or a single endoscope conjugated with a transabdominal trocar, Park et al. [8] and Swanstrom et al. [9] experienced significant difficulties performing cholecystectomy using shape-lock technology. These authors reported difficulties related to controlling the pneumoperitoneum and obtaining a stable platform for anatomy exposure, organ retraction, secure grasping, and adequate triangulation of instruments. Rolanda et al. [10] introduced the concept of combined approaches by natural orifice using a combined transgastric and transvesical approach for cholecystectomy. The authors overcame most of the limitations previously reported for those who attempted to perform cholecystectomy exclusively through a transgastric approach. Pneumoperitoneum was controlled by ureteroscope, and the working channel of this device was used to pass instruments for gallbladder grasping and manipulation, overcoming the lack of triangulation. Subsequently, the gallbladder was removed transorally, but the gastrotomy site was able to be closed.

In a nonsurvival study, Lima et al. [11] expanded the concept of combined transgastric and transvesical approaches performing nephrectomy in female pigs. Under ureteroscope visualization through a 5-mm transvesical port, researchers controlled the orally introduced flexible gastroscope by the gastrotomy into the peritoneal cavity. Right or left nephrectomy was carried out using instruments introduced by devices that worked in the renal hilum, alternating intervention on dissection or retraction procedures. In all animals, both kidneys were visualized, and the renal vessels and ureter were reasonably individualized and ligated separately with ultrasonic scissors introduced through the transvesical port. In two early cases, mild hemorrhage occurred after ultrasonic ligation. Thus, complete renal release and mobilization to the stomach were achieved in all animals, but the gastrotomy site could not be 
closed. The authors also reported that additional improvements are needed with better devices and instruments.

Recently, Sawyer et al. [12] described a partial cystectomy by intravesical transurethral techniques in a porcine model. An endoscopic loop device was advanced through one port of the multichannel cystoscope. Through the second port, a flexible toothed grasping device was advanced through the loop to grasp the targeted area of the bladder wall. Then the grasper was slowly withdrawn while maintaining a grip on the "pseudotumor" through the loop. A full-thickness bladder segment was then excised using cutting current. At the end of the procedure, the specimen was removed en bloc with the cystoscope, and the bladder wall defect was reapproximated with endoscopic clips. The authors reported that further investigation in chronic models will be required to determine the potential for safe adaptation to human beings.

In a human case, Gettman et al. [13] performed a transvesical peritoneoscopy using a ureteroscope prior to performing a robot-assisted radical prostatectomy. They used the same technical mode of transvesical approach in a porcine model with few modifications (e.g., instead of a ureteral catheter, the authors used a balloon dilator).

In 2009, Lima et al. [6] described the transvesical approach to the peritoneal cavity, noting that closure of a 5 -mm bladder hole is not absolutely necessary if bladder drainage is assured. The development of an effective closure device might enable the widespread adoption of transvesical port in NOTES. This was the rationale for this research group to report the usefulness of T-fasteners with a locking cinch system. They demonstrated the feasibility and the safety of endoscopic closure of vesical perforations with an endoscopic suturing kit (T-fasteners with a locking cinch) in a survival porcine model. Three steps were involved in the endoscopic closure of the perforation: 1) With the animals in the Trendelenburg position, the needle punctured on the edge of the perforation (the 19-gauge needle was loaded with the metal T-tag attached to a 3.0 violet Monocryl $90-\mathrm{cm}$ thread and was advanced through the working channel of the cystoscope and placed through the full-thickness of one edge of the bladder wall). By advancing the stylet, the T-tag and thread were released from the needle and left in the exterior part of the bladder; 2) needle puncture of the opposite edge of the perforation, followed by release of the T-tag, which was performed in a similar way; and 3) knot tying was then accomplished, followed by suture cutting with a lock-and-cut combination device, which was advanced to tie the threads together. The defect was closed by pulling the threads on either side of the incision together until they were snug against the lock, and then by closing the lock and subsequently cutting the threads with the combination thread-locking and suturecutting device. This resulted in a secure closure of the perforation. No catheter was left in the bladder. All animals were evaluated daily; the postmortem examination 15 days after surgery revealed complete healing of the bladder wall incision. The authors concluded that these findings provided immediate support for clinical application of this method to close bladder perforations both in management of bladder rupture and transvesical port in NOTES procedures.

More recently, Metzelder et al. [14] closed the bladder perforations after five nephroureterectomies with an endoloop via a $15-\mathrm{mm}$ umbilical trocar with the assistance of a 2-mm transurethrally placed endoscopic clamp.

\section{Advantages and Disadvantages of Transvesical Port}

Lower abdominal access points, including the transvesical, transvaginal, and transcolonic ports, can be used as an isolated or complementary transgastric port. Several researchers who performed complex abdominal procedures via an isolated transgastric pathway have encountered problems with decreased triangulation and retroflexion [15]. Lower abdominal ports, however, may overcome some of these limitations, with added advantage in complex operations. Although lower abdominal ports provide frontal access to upper abdominal organs and enable improved instrument access, the transvesical port offers sterility and the anatomical advantage of the most anterior lower abdominal access. Further, the transvesical port enables rigid instrument introduction and does not necessarily require closure. Accessing the peritoneal cavity through a natural orifice risks damaging adjacent organs during visceral wall incision. Among natural orifice ports, transvesical port creation with a Seldinger-like technique may be the safest approach because bowel loops that contact the bladder wall are unrestricted in the abdomen, which make them run away from bladder instruments. Further, in procedures involving two natural orifices, such as transgastric and transvesical, the transvesical image can easily monitor the transgastric creation.

The transvesical port procedure involves a significant challenge related to instrument size, limiting organ retrieval through this port. Another concern related to transvesical port use is the necessity of bladder closure. Vesical perforation involves potential complications, such as peritoneal urine leakage with secondary infection (peritonitis), that commonly occur as delayed complications of undiagnosed traumatic vesical perforation or pathological bladder conditions, such as neoplasms. Experiments in pigs demonstrated that $5-\mathrm{mm}$ bladder hole closure is not absolutely necessary if bladder drainage is assured. However, this limitation might be overcome with the recent development of an effective closure method of bladder perforations. In fact, the feasibility and safety of an 
endoscopic suturing kit (T-fasteners with a locking cinch) application in vesical perforations was demonstrated in a survival porcine model.

\section{Potential Applications}

Given its anatomic and physiological properties, we anticipate that transvesical port in humans will be useful for the following: simple abdominal procedures; peritoneoscopy; varicocelectomy; tubal ligation; renal cyst marsupialization; and procedures in the diaphragm. Moreover, we demonstrated the usefulness of the transvesical port as an accessory port: pure combined NOTES complex procedures; cholecystectomy; and nephrectomy.

\section{Conclusions}

The transvesical approach broke a classical sanctuary of urology: the wall of the bladder. The transvesical port is sterile, easy, and safe to create. It can be closed, leaving no bladder catheter. There is enough experimental support to apply these concepts in humans.

Disclosure No potential conflicts of interest relevant to this article were reported.

\section{References}

Papers of particular interest, published recently, have been highlighted as:

•- Of major importance

1. •- Rattner D, Kalloo A, ASGE/SAGES Working Group: ASGE/ SAGES working group on natural orifice translumenal endoscopic surgery. Surg Endosc 2006, 20:329-333. Leaders from the American Society of Gastrointestinal Endoscopy and the Society of American Gastrointestinal and Endoscopic Surgeons agreed that translumenal endoscopic surgery could offer significant benefits to patients, such as less pain, faster recovery, and better cosmesis, than current laparoscopic techniques. The group identified the barriers that needed to be surmounted for the development of translumenal endoscopic intraperitoneal surgery and developed a list of next steps and guidelines to move this concept ahead.

2. • Kalloo AN, Singh VK, Jagannath SB, et al.: Flexible transgastric peritoneoscopy: a novel approach to diagnostic and therapeutic interventions in the peritoneal cavity. Gastrointest Endosc 2004, 60:114-117. In this article, the authors described a new port to the peritoneal cavity through a transgastric port in a porcine model.

3. Lima E, Rolanda C, Correia-Pinto J: NOTES performed using multiple ports of entry: current experience and potential implications for urologic applications. J Endourol 2009, 23:759-764.

4. Harrell AG, Heniford BT: Minimally invasive abdominal surgery: lux et veritas past, present, and future. Am J Surg 2005, 190:239243.

5. •- Lima E, Rolanda C, Pêgo JM, et al.: Transvesical endoscopic peritoneoscopy: a novel $5 \mathrm{~mm}$ port for intra-abdominal scarless surgery. J Urol 2006, 176:802-805. This was the first study that demonstrated the feasibility and safety of transvesical port to the peritoneal cavity in a porcine model.

6. Lima E, Rolando C, Osório L, et al.: Endoscopic closure of transmural bladder wall perforations. Eur Urol 2009, 56:151-157.

7. Lima E, Henriques-Coelho T, Rolanda C, et al.: Transvesical thoracoscopy: a natural orifice translumenal endoscopic approach for thoracic surgery. Surg Endosc 2007, 21:854-858.

8. Park PO, Bergström M, Ikeda K, et al.: Experimental studies of transgastric gallbladder surgery: cholecystectomy and cholecystogastric anastomosis (videos). Gastrointest Endosc 2005, 61:601606.

9. Swanstrom LL, Kozarek R, Pasricha PJ, et al.: Development of a new access device for transgastric surgery. J Gastrointest Surg 2005, 9:1129-1137.

10. Rolanda C, Lima E, Pêgo JM, et al.: Third-generation cholecystectomy by natural orifices: transgastric and transvesical combined approach (with video). Gastrointest Endosc 2007, 65:111-117.

11. Lima E, Rolanda C, Pêgo JM, et al.: Third-generation nephrectomy by natural orifice translumenal endoscopic surgery. J Urol 2007, 178:2648-2654.

12. Sawyer MD, Cherullo EE, Elmunzer BJ, et al.: Pure natural orifice translumenal endoscopic surgery partial cystectomy: intravesical transurethral and extravesical transgastric techniques in a porcine model. Urology 2009, 74:1049-1053.

13. Gettman MT, Lotan Y, Napper CA, Cadeddu JA: Transvaginal laparoscopic nephrectomy: development and feasibility in the porcine model. Urology 2002, 59:446-450.

14. Metzelder M, Vieten G, Goseman JH, et al.: Endoloop closure of the urinary bladder is safe and efficient in female piglets undergoing transurethral NOTES nephrectomy. Eur J Pediatr Surg 2009, 19:362-365.

15. Rolanda C, Lima E, Silva D, et al.: In vivo assessment of gastrotomy closure with over-the-scope clips in an experimental model for varicocelectomy (with video). Gastrointest Endosc 2009, 70:1137-1145. 